Arch. Math. 70 (1998) 57-65

0003-889X/98/010057-09 \$3.30/0

(C) Birkhäuser Verlag, Basel, 1998

Archiv der Mathematik

\title{
Strictly singular operators and isomorphisms of Cartesian products of power series spaces
}

\author{
By
}

P. B. DJAKov*), S. Önal, T. TerzioĞLu and M. Yurdakul

\begin{abstract}
V. P. Zahariuta, in 1973, used the theory of Fredholm operators to develop a method to classify Cartesian products of locally convex spaces. In this work we modify his method to study the isomorphic classification of Cartesian products of the kind $E_{0}^{p}(a) \times E_{\infty}^{q}(b)$ where $1 \leqq p, q<\infty, p \neq q, a=\left(a_{n}\right)_{n=1}^{\infty}$ and $b=\left(b_{n}\right)_{n=1}^{\infty}$ are sequences of positive numbers and $E_{0}^{p}(a), E_{\infty}^{q}(b)$ are respectively $\ell^{p}$-finite and $\ell^{q}$-infinite type power series spaces.
\end{abstract}

Introduction. Let $\left(a_{i k}\right)_{i, k \in \mathbb{N}}$ be a matrix of real numbers, such that $0 \leqq a_{i k} \leqq a_{i, k+1}$ for all $i, k$ and $p \geqq 1$. We denote by $K^{p}\left(a_{i k}\right)$ the $\ell^{p}$-Köthe space defined by the matrix $\left(a_{i k}\right)$, i.e. the space of all sequences of scalars $x=\left(x_{i}\right)$ such that

$$
|x|_{k}:=\left(\sum_{i}\left(\left|x_{i}\right| a_{i k}\right)^{p}\right)^{1 / p}<\infty \quad \forall k \in \mathbb{N} .
$$

With the topology generated by the system of seminorms $\left\{|\cdot|_{k}, k \in \mathbb{N}\right\}$ it is a Fréchet space. If $a=\left(a_{i}\right)$ is a sequence of positive numbers the Köthe spaces

$$
E_{0}^{p}(a)=K^{p}\left(\exp \left(-\frac{1}{k} a_{i}\right)\right), \quad E_{\infty}^{p}(a)=K^{p}\left(\exp \left(k a_{i}\right)\right)
$$

are called, respectively, $\ell^{p}$-finite and $\ell^{p}$-infinite type power series spaces. They are Schwartz spaces if and only if $a_{i} \rightarrow \infty$.

Power series spaces play an important role in Functional Analysis because they provide sequence space representations for large classes of spaces of (analytic or $\mathbb{C}^{\infty}$ ) functions (see for more details $[8,9,14])$. Their isomorphic classification and structure properties were studied by Kolmogorov, Pelczynski, Mityagin and many other mathematicians and the question of isomorphic classification was solved completely in the Schwartzian case (see [8] for details) by the help of classical linear topological invariants, namely approximative and diametral dimensions.

For arbitrary (non-Schwartzian) spaces, Mityagin [10] obtained a complete isomorphic classification of $\ell^{2}$-power series spaces (see also [12]). Moreover, he initiated in this paper a

Mathematics Subject Classification (1991): 46A04, 47A99.

*) Research supported by TÜBITAK-NATO Fellowship Program and partially by NRF of Bulgaria, grant MM-409/94. 
method to construct new (generalized) linear topological invariants that are more powerful than approximative and diametral dimensions. Zahariuta [18] developed this method for Köthe spaces and obtained new results about isomorphic classification for some classes of Köthe spaces that include Cartesian and tensor products of power series spaces (for further developments see the survey [22]).

Another approach to the isomorphic classification of Cartesian products was Zahariuta's use of the theory of Fredholm operators [19, 20]. We modify Zahariuta's method (following [13], see also [21]) in order to extend its area of applications, and use the modified version to study the isomorphic classification of Cartesian products of the kind $E_{0}^{p}(a) \times E_{\infty}^{q}(b)$, where $a, b$ are sequences of positive numbers and $p, q \in[1, \infty)$. Let us note that in [19], [20] a complete isomorphic classification of these spaces is obtained in the case where at least one of the sequences $a, b$ tends to $\infty$ (i.e. at least one of the Cartesian factors is a Schwartz space). On the other hand, in the non-Schwartzian case a complete isomorphic classification of the spaces $E_{0}^{1}(a) \times E_{\infty}^{1}(b)$ is obtained in [2], [3] by using the appropriate linear topological invariants. In the same way one can characterize the isomorphisms of the spaces $E_{0}^{p}(a) \times E_{\infty}^{p}(b)$, where $p$ is fixed, $p \in[1, \infty)$. Here we complete these results by studying the non-Schwartzian case for $p \neq q$.

Some of our results are presented without proof in [4].

Acknowledgement. We would like to thank professors S. L. Troyanski and V. P. Zahariuta for helpful discussions concerning the proof of Proposition 4.

Preliminaries. Let $X$ and $Y$ be locally convex spaces and $T: X \rightarrow Y$ be a continuous linear operator. The operator $T$ is bounded (respectively precompact) if there exists a neighborhood $U$ of zero in $X$ such that $T(U)$ is bounded (respectively precompact) in $Y$. The operator $T$ is strictly singular if its restriction on any closed infinite-dimensional subspace of $X$ is not an isomorphism.

We write $(X, Y) \in \mathscr{B},(X, Y) \in \mathscr{K},(X, Y) \in \mathscr{S} \mathscr{S},(X, Y) \in \mathscr{B} \mathscr{S} \mathscr{S}$ if every continuous linear operator from $X$ into $Y$ is bounded, precompact, strictly singular, bounded and strictly singular, respectively. Since every precompact operator is bounded and strictly singular the relation $(X, Y) \in \mathscr{K}$ implies $(X, Y) \in \mathscr{B} \mathscr{S} \mathscr{S}$. The converse is not true. For example, if $1 \leqq p<q<\infty$ then $\left(\ell^{p}, \ell^{q}\right) \in \mathscr{B} \mathscr{S} \mathscr{S}$, but $\left(\ell^{p}, \ell^{q}\right) \notin \mathscr{K}$ since the identity mapping from $\ell^{p}$ to $\ell^{q}$ is not compact (see [7], Vol. I, Ch. 2. Sect. C).

A Köthe matrix $\left(a_{i k}\right)$ is of type $\left(d_{1}\right)$ or $\left(d_{2}\right)$, respectively, if the following condition holds:

$$
\begin{aligned}
& \exists k_{0} \forall k \exists m, C: \quad a_{i k}^{2} \leqq C a_{i k_{0}} a_{i m} ; \\
& \forall k \exists m \forall \ell \exists C: \quad C a_{i m}^{2} \geqq a_{i k} a_{i \ell} .
\end{aligned}
$$

The corresponding Köthe spaces are referred as $\left(\mathrm{d}_{1}\right)$ or $\left(\mathrm{d}_{2}\right)$ spaces. It is easy to see that finite (respectively infinite) power series spaces are $\left(\mathrm{d}_{2}\right)$ (respectively $\left(\mathrm{d}_{1}\right)$ ) spaces. V. P. Zahariuta [20] showed that $(X, Y) \in \mathscr{B}$ if $X$ and $Y$ are locally convex spaces with absolute bases, satisfying the conditions $\left(\mathrm{d}_{2}\right)$ and $\left(\mathrm{d}_{1}\right)$ respectively. Of course then $(X, Y) \in \mathscr{K}$ if $X$ is a Schwartz space or $Y$ is a Montel space. D. Vogt [15] studied the relation $(X, Y) \in \mathscr{B}$ for Fréchet spaces. Using his results ( Satz 6.2 and Prop. 5.3 in [15]), one obtains that $(X, Y) \in \mathscr{B}$ 
if $X$ and $Y$ are Fréchet spaces satisfying the conditions $(\bar{\Omega})$ and $(D N)$ respectively. One can easily see that $\left(\mathrm{d}_{2}\right) \Rightarrow(\bar{\Omega})$ and $\left(\mathrm{d}_{1}\right) \Rightarrow(D N)$, so the following statement holds.

Proposition 1. If $X$ is $\left(\mathrm{d}_{2}\right)$-Köthe space and $Y$ is $\left(\mathrm{d}_{1}\right)$-Köthe space then $(X, Y) \in \mathscr{B}$. In particular, for any $p, q \in[1, \infty)$ we have $\left(E_{0}^{p}(a), E_{\infty}^{q}(b)\right) \in \mathscr{B}$.

A locally convex space $X$ is called Mackey-complete if for every bounded, absolutely convex, closed subset $A \subset X$ the linear span of $A$ is a Banach space with unit ball $A$. It is easy to see that a sequentially complete locally convex space is Mackey-complete. In particular every Fréchet space is Mackey-complete.

An operator acting between two linear topological spaces is Fredholm if it is an open mapping with finite dimensional kernel and finite codimensional closed range. An operator $T$ acting in a linear topological space $X$ will be called Riesz type operator if $1_{X}-T$ is a Fredholm operator. The following proposition is due to V. V. Wrobel (see [16], Th. 3 and [17], Satz 1):

Proposition 2. Bounded strictly singular operators between Mackey-complete spaces form an ideal of Riesz type operators.

If $E$ and $\tilde{E}$ are linear topological spaces with bases $\left(e_{i}\right)$ and $\left(\tilde{e}_{j}\right)$ respectively, then an operator $T: E \rightarrow \tilde{E}$ is quasidiagonal if there exist scalars $r_{i}$ and a mapping $j(i)$ such that $T e_{i}=r_{i} \tilde{e}_{j(i)} \forall i$. The spaces $E$ and $\tilde{E}$ are quasidiagonally isomorphic (we write $E \stackrel{q d}{\cong} \tilde{E}$ ) if there exists an isomorphism $T: E \rightarrow \tilde{E}$ that is quasidiagonal.

Let $K^{p}\left(a_{i k}\right)$ be a $\ell^{p}$-Köthe space, $1 \leqq p<\infty$. For any strictly increasing sequence of integers $(j(i))$ the Köthe space $K^{p}\left(a_{j(i) k}\right)$ is called a basic subspace of $K^{p}\left(a_{i k}\right)$. Obviously each basic subspace is complemented. C. Bessaga [1] made the following conjecture:

If $X$ is a (nuclear) Köthe space then every complemented subspace of $X$ with basis is quasidiagonally isomorphic to a basic subspace of $X$.

Modifying some ideas of M. Dragilev [5], C. Bessaga [1] proved his conjecture for stable nuclear finite or infinite power series spaces. Later many authors worked on this subject and Bessaga's conjecture was proved for wide classes of spaces. In particular, for power series spaces the following proposition holds (see $[10,11]$ and $[6]$ ).

Proposition 3. If $X$ is $\ell^{p}$-finite (respectively $\ell^{p}$-infinite) power series space then every complemented subspace of $X$ with $\ell^{p}$-absolute basis is quasi-diagonally isomorphic to a basic subspace of $X$.

Finally we consider the following

Proposition 4. If $1 \leqq p<q<\infty$ and $K^{p}\left(a_{i k}\right) \cong K^{q}\left(b_{i k}\right)$ then the space $K^{p}\left(a_{i k}\right)$ is nuclear.

Proof. We consider two cases: $p<2$ and $p \geqq 2$.

Let $p<2$. It is easy to see that the space $K^{p}\left(a_{i k}\right)$ is nuclear if and only if

$$
\exists r \forall k \exists m: \quad \sum_{i=1}^{\infty}\left(\frac{a_{i k}}{a_{i m}}\right)^{r}<\infty .
$$


Since $\left(\ell^{q}, \ell^{p}\right) \in \mathscr{K}$ the space $K^{p}\left(a_{i k}\right)$ is Schwartzian. Let $T: K^{p}\left(a_{i k}\right) \rightarrow K^{q}\left(b_{i k}\right)$ be an isomorphism. Then we have

$$
\forall k \exists k_{1}, m, C_{1}, C_{2}:|x|_{k} \leqq C_{1}|T x|_{k_{1}} \leqq C_{2}|x|_{m} .
$$

Choose $m$ big enough so that $a_{i k} / a_{i m} \rightarrow 0$ (it is possible since the space is Schwartzian). We can assume without loss of generality that the sequence $\left(a_{i k} / a_{i m}\right)$ is decreasing (if not, one can reorder it).

Using the fact that the space $\ell^{q}$ is of type $q_{1}=\min (2, q)$ (see [7], Vol. 2, p.72) we obtain, for any $n$, a $\theta_{i}, i=1, \ldots, n, \theta_{i}=1$ or $\theta_{i}=-1$ such that

$$
\begin{aligned}
\frac{a_{n k}}{a_{n m}} n^{1 / p} & \leqq\left(\sum_{i=1}^{n}\left(\frac{a_{i k}}{a_{i m}}\right)^{p}\right)^{1 / p}=\left|\sum_{i=1}^{n} \theta_{i} \frac{e_{i}}{a_{i m}}\right|_{k} \leqq C_{1}\left|\sum_{i=1}^{n} \theta_{i} \frac{T e_{i}}{a_{i m}}\right|_{k_{1}} \\
& \leqq M C_{1}\left(\sum_{i=1}^{n}\left(\frac{\left|T e_{i}\right|_{k_{1}}}{a_{i m}}\right)^{q_{1}}\right)^{1 / q_{1}} \leqq M C_{2} n^{1 / q_{1}},
\end{aligned}
$$

where $M$ is a constant. From here it follows that

$$
\frac{a_{n k}}{a_{n m}} \leqq M C_{2} n^{\frac{1}{q_{1}}-\frac{1}{p}}
$$

therefore the sequence $\left(a_{i k} / a_{i m}\right)$ belongs to $\ell^{r}$ for $r>p q_{1} /\left(q_{1}-p\right)$.

In the case $p \geqq 2$ we show that the space $K^{q}\left(b_{i k}\right)$ is nuclear using the fact that $\ell^{p}$ has cotype $p$. Since $T^{-1}$ is an isomorphism we have

$$
\forall k \exists k_{1}, m, C_{1}, C_{2}:|x|_{k} \leqq C_{1}\left|T^{-1} x\right|_{k_{1}} \leqq C_{2}|x|_{m} .
$$

As in the first case, we can assume that the sequence $\left(b_{i k} / b_{i m}\right)$ is decreasing, and we obtain

$$
\begin{aligned}
\frac{b_{n k}}{b_{n m}} n^{1 / p} & \leqq\left(\sum_{i=1}^{n}\left(\frac{b_{i k}}{b_{i m}}\right)^{p}\right)^{1 / p}=\left(\sum_{i=1}^{n}\left|\frac{e_{i}}{b_{i m}}\right|_{k}^{p}\right)^{1 / p} \leqq C_{1}\left(\sum_{i=1}^{n}\left|\frac{T^{-1} e_{i}}{b_{i m}}\right|_{k_{1}}^{p}\right)^{1 / p} \\
& \leqq M C_{1}\left|\sum_{i=1}^{n} \theta_{i} \frac{T^{-1} e_{i}}{b_{i m}}\right|_{k_{1}} \leqq M C_{2}\left|\sum_{i=1}^{n} \theta_{i} \frac{e_{i}}{b_{i m}}\right|_{m}=M C_{2} n^{1 / q}
\end{aligned}
$$

From here the nuclearity follows as in the first case.

Modification of Zahariuta's method. We present now the modification of the method for isomorphic classification developed by Zahariuta in [20]. As usual we identify an operator $T=\left(T_{i j}\right): E_{1} \times E_{2} \rightarrow F_{1} \times F_{2}$ with the corresponding $2 \times 2$-matrix, whose entries are operators acting between the factors of the Cartesian products.

Lemma 1. If $T=\left(T_{i j}\right): E_{1} \times E_{2} \rightarrow F_{1} \times F_{2}$ is an isomorphism such that $T_{11}: E_{1} \rightarrow F_{1}$ is also an isomorphism then $E_{2} \cong F_{2}$.

Proof. Let $T^{-1}=\left(S_{i j}\right)$. Consider the operators

$$
S_{22}: F_{2} \rightarrow E_{2}, \quad H: E_{2} \rightarrow F_{2},
$$

where $H=T_{22}-T_{21} T_{11}^{-1} T_{12}$. Taking into account the fact that $T_{11} S_{12}+T_{12} S_{22}=0$ we obtain

$$
H S_{22}=T_{22} S_{22}-T_{21} T_{11}^{-1} T_{12} S_{22}=T_{22} S_{22}+T_{21} S_{12}=1_{F_{2}} .
$$


In an analogous way from $S_{21} T_{11}+S_{22} T_{21}=0$ it follows that

$$
S_{22} T_{22}-S_{22} T_{21} T_{11}^{-1} T_{12}=S_{22} T_{22}+S_{21} T_{12}=1_{E_{2}} .
$$

Hence the spaces $E_{2}$ and $F_{2}$ are isomorphic.

Theorem 1. If $X_{1}, X_{2}, \tilde{X}_{1}, \tilde{X}_{2}$ are linear topological spaces such that $X_{1} \times X_{2} \cong \tilde{X}_{1} \times \tilde{X}_{2}$ and each operator acting in $X_{1}$ that factors over $\tilde{X}_{2}$ is Riesz type operator then there exist a finite dimensional subspace $L_{1}$ in $X_{1}$ and complemented subspaces $E_{1} \subset X_{1}$ and $M_{1} \subset \tilde{X}_{1}$ such that

$$
X_{1} \cong E_{1} \times L_{1}, \quad \tilde{X}_{1} \cong E_{1} \times M_{1}, \quad M_{1} \times \tilde{X}_{2} \cong L_{1} \times X_{2} .
$$

Proof. Let $T=\left(T_{i j}\right): X_{1} \times X_{2} \rightarrow \tilde{X}_{1} \times \tilde{X}_{2}$ be an isomorphism, and let $T^{-1}=\left(S_{i j}\right)$. Then we have $S_{11} T_{11}+S_{12} T_{21}=1_{X_{1}}$, and since $S_{12} T_{21}$ is Riesz type operator $S_{11} T_{11}$ is a Fredholm operator. Thus the subspace $L_{1}:=\operatorname{ker} S_{11} T_{11}$ has finite dimension, the subspace $G=S_{11} T_{11}\left(X_{1}\right)$ is closed and has finite codimension. Let $E_{1}$ be a complementary subspace of $L_{1}$ in $X_{1}$ and $\pi_{G}$ be a projection on $G$. Obviously the operator $A=S_{11} T_{11}: E_{1} \rightarrow G$ is an isomorphism. We set $F_{1}=T_{11}\left(E_{1}\right)$; then $T_{11}$ maps $E_{1}$ into $F_{1}$ isomorphically. Moreover $F_{1}$ is a complemented subspace of $\tilde{X}_{1}$. Indeed, it is easy to see that the operator

$$
P=T_{11} A^{-1} \pi_{G} S_{11}: \tilde{X}_{1} \rightarrow \tilde{X}_{1}
$$

is a projection on $F_{1}$.

Let $M_{1}=P^{-1}(0)$ be the corresponding complementary subspace. Then we have

$$
X_{1} \cong E_{1} \times L_{1}, \quad \tilde{X}_{1}=F_{1} \oplus M_{1} \cong E_{1} \times M_{1},
$$

so applying the lemma to $E_{1} \times\left(L_{1} \times X_{2}\right)$ and $F_{1} \times\left(M_{1} \times \tilde{X}_{2}\right)$ we obtain $L_{1} \times X_{2} \cong M_{1} \times \tilde{X}_{2}$.

For any locally convex space $X$ and any integer $s$, the symbol $X^{(s)}$ denotes an $s$ codimensional subspace of $X$ if $s \geqq 0$ and a product of the kind $X \times L$, where $\operatorname{dim} L=-s$, if $s<0$.

Corollary 1 (see [20], §6). Retaining the assumptions of the theorem, if each operator acting in $\tilde{X}_{1}$ that factors over $X_{2}$ is Riesz type operator, then the subspace $M_{1}$ has finite dimension, so $\tilde{X}_{1} \cong X_{1}^{(s)}, \tilde{X}_{2} \cong X_{2}^{(-s)}$ with $s=\operatorname{dim} L_{1}-\operatorname{dim} M_{1}$.

Proof. By the assumption it follows immediately that each operator acting in $M_{1}$ that factors over $X_{2}$ is Riesz type operator. Applying the theorem to the isomorphism $M_{1} \times \tilde{X}_{2} \cong L_{1} \times X_{2}$ we see that there exist complementary subspaces $M_{2}$ and $M_{3}$ of $M_{1}$ such that $M_{3}$ is finite dimensional and $M_{2}$ is isomorphic to a complemented subspace of $L_{1}$, hence $M_{1}$ has finite dimension.

Isomorphisms of Cartesian products of power series spaces. We begin with the following lemma.

Lemma 2. If $X=\operatorname{proj}_{k} X_{k}$ and $Y=\operatorname{proj}_{m} Y_{m}$ are projective limits of normed spaces such that $\forall k, m\left(X_{k}, Y_{m}\right) \in \mathscr{S} \mathscr{S}$ then each bounded operator $T: X \rightarrow Y$ is strictly singular. 
Proof. Suppose, on the contrary, there is a bounded operator $T: X \rightarrow Y$ that is not strictly singular. Then there exists an infinite-dimensional subspace $M \subset X$ such that the restriction $\left.T\right|_{M}$ is an isomorphism. This means in particular that

$$
\forall k \exists m(k), C_{k}:|x|_{k} \leqq C_{k}|T x|_{m(k)} \quad \forall x \in M .
$$

On the other hand, since $T$ is bounded, we have

$$
\exists k_{0} \forall m \exists D_{m}:|T x|_{m} \leqq D_{m}|x|_{k_{0}} \quad \forall x \in X,
$$

therefore

$$
|x|_{k_{0}} \leqq C_{k_{0}}|T x|_{m\left(k_{0}\right)} \leqq C_{k_{0}} D_{m\left(k_{0}\right)}|x|_{k_{0}} \quad \forall x \in M,
$$

i.e., if we consider $T$ as an operator from $X_{k_{0}}$ to $Y_{m\left(k_{0}\right)}$ then its restriction to $M$ is an isomorphism. This contradicts the assumption $\left(X_{k_{0}}, Y_{m\left(k_{0}\right)}\right) \in \mathscr{S} \mathscr{S}$, so the lemma is proved.

Theorem 2. Let $p \neq \tilde{q}, q \neq \tilde{p}, 1 \leqq p, q, \tilde{p}, \tilde{q}<\infty$, and $a, b, \tilde{a}, \tilde{b}$ be sequences of positive numbers. Then the following conditions are equivalent:

(i) $E_{0}^{p}(a) \times E_{\infty}^{q}(b) \cong E_{0}^{\tilde{p}}(\tilde{a}) \times E_{\infty}^{\tilde{q}}(\tilde{b})$;

(ii) there exists an integer such that

$$
E_{0}^{\tilde{p}}(\tilde{a}) \cong\left(E_{0}^{p}(a)\right)^{(s)} \quad \text { and } \quad E_{\infty}^{\tilde{q}}(\tilde{b}) \cong\left(E_{\infty}^{q}(b)\right)^{(-s)} .
$$

Proof. By Proposition 1

$$
\left(E_{0}^{p}(a), E_{\infty}^{\tilde{q}}(\tilde{b})\right) \in \mathscr{B} \quad \text { and } \quad\left(E_{0}^{\tilde{p}}(\tilde{a}), E_{\infty}^{q}(b)\right) \in \mathscr{B} .
$$

We apply Lemma 2 to the pairs of spaces

$$
\left.E_{0}^{p}(a)=\operatorname{proj}_{k} \ell^{p}\left(\exp \left(-\frac{1}{k} a_{n}\right)\right), \quad E_{\infty}^{\tilde{q}}(\tilde{b})=\operatorname{proj}_{k} \ell^{\tilde{q}}\left(\exp k \tilde{b}_{n}\right)\right)
$$

and $E_{0}^{\tilde{p}}(\tilde{a}), E_{\infty}^{q}(b)$.

Since $\left(\ell^{p}, \ell^{q}\right) \in \mathscr{S} \mathscr{S}$ for $p<q$ and $\left(\ell^{p}, \ell^{q}\right) \in \mathscr{K}$ for $p>q$ (see [7], Vol. I, Ch. 2, Sect. C) the assumptions of Lemma 2 are fulfilled, hence

$$
\left(E_{0}^{p}(a), E_{\infty}^{\tilde{q}}(\tilde{b})\right) \in \mathscr{B} \mathscr{S} \mathscr{S} \quad \text { and } \quad\left(E_{0}^{\tilde{p}}(\tilde{a}), E_{\infty}^{q}(b)\right) \in \mathscr{B} \mathscr{S} \mathscr{S} .
$$

Now Corollary 1 completes the proof.

Corollary 2. Under the assumptions of the theorem it follows from Proposition 4 that:

if $p \neq \tilde{p}$ the spaces $E_{0}^{p}(a), E_{0}^{\tilde{p}}(\tilde{a})$ are nuclear;

if $q \neq \tilde{q}$ the spaces $E_{\infty}^{q}(b), E_{\infty}^{\tilde{q}}(\tilde{b})$ are nuclear.

In the next theorem we consider the case when one of the conditions $p \neq \tilde{q}, q \neq \tilde{p}$ does not hold.

Theorem 3. Let $a, b, \tilde{a}, \tilde{b}$ be sequences of positive numbers and $p, q, \tilde{q} \in[1, \infty), p \neq \tilde{q}$. Then

(i) $E_{0}^{p}(a) \times E_{\infty}^{q}(b) \cong E_{0}^{q}(\tilde{a}) \times E_{\infty}^{\tilde{q}}(\tilde{b})$; 
if and only if

(A) when $\tilde{a}$ or $b$ tends to $\infty$, there exists an integer $s$ such that

$$
E_{0}^{q}(\tilde{a}) \cong\left(E_{0}^{p}(a)\right)^{(s)} \quad \text { and } \quad E_{\infty}^{\tilde{q}}(\tilde{b}) \cong\left(E_{\infty}^{q}(b)\right)^{(-s)} ;
$$

(B) when non tends to $\infty$, there exist complementary subsequences $\tilde{a}^{\prime}, \tilde{a}^{\prime \prime}$ of $\tilde{a}$ and $b^{\prime}, b^{\prime \prime}$ of $b$ such that $\tilde{a}^{\prime \prime}, b^{\prime \prime}$ are bounded and, further,

$$
E_{0}^{p}(a) \cong E_{0}^{q}\left(\tilde{a}^{\prime}\right) \quad \text { and } \quad E_{\infty}^{\tilde{q}}(\tilde{b}) \cong E_{\infty}^{q}\left(b^{\prime}\right) .
$$

Proof. As in Theorem 2 it follows

$$
\left(E_{0}^{p}(a), E_{\infty}^{\tilde{q}}(\tilde{b})\right) \in \mathscr{B} \mathscr{S} \mathscr{S} \quad \text { and } \quad\left(E_{0}^{q}(\tilde{a}), E_{\infty}^{q}(b)\right) \in \mathscr{B} .
$$

In the case $(\mathrm{A})$ at least one of the spaces $E_{0}^{q}(\tilde{a}), E_{\infty}^{q}(b)$ is Schwartzian, therefore

$$
\left(E_{0}^{q}(\tilde{a}), E_{\infty}^{q}(b)\right) \in \mathscr{K} \subset \mathscr{B} \mathscr{S} \mathscr{S} .
$$

Thus the result follows from Corollary 1.

In the case (B) we have, by Theorem 1 , a finite codimensional subspace of $E_{0}^{p}(a)$ is isomorphic to a complemented subspace of $E_{0}^{q}(\tilde{a})$. Since $\tilde{a}_{i} \rightarrow \infty$ the space $E_{0}^{q}(\tilde{a})$ contains a basic subspace isomorphic to $\ell^{q}$. Since any finite codimensional subspace of $\ell^{q}$ is isomorphic to $\ell^{q}$, then $E_{0}^{p}(a)$ is isomorphic to a complemented subspace of $E_{0}^{q}(\tilde{a})$. By Proposition 3 there exist complementary subsequences $\tilde{a}^{\prime}, \tilde{a}^{\prime \prime}$ of the sequence $\tilde{a}$ such that

$$
E_{0}^{q}(\tilde{a})=E_{0}^{q}\left(\tilde{a}^{\prime}\right) \oplus E_{0}^{q}\left(\tilde{a}^{\prime \prime}\right) \text { and } E_{0}^{p}(a) \cong E_{0}^{q}\left(\tilde{a}^{\prime}\right) .
$$

From Theorem 1 it follows that $E_{\infty}^{q}(b) \cong E_{0}^{q}\left(\tilde{a}^{\prime \prime}\right) \times E_{\infty}^{\tilde{q}}(\tilde{b})$, so again by Proposition 3 there exist complementary subsequences $b^{\prime}, b^{\prime \prime}$ of the sequence $b$ such that

$$
E_{\infty}^{\tilde{q}}(\tilde{b}) \cong E_{\infty}^{q}\left(b^{\prime}\right) \text { and } E_{0}^{q}\left(\tilde{a}^{\prime \prime}\right) \cong E_{\infty}^{q}\left(b^{\prime \prime}\right) .
$$

It is easy to see that the subsequences $\tilde{a}^{\prime \prime}, b^{\prime \prime}$ are bounded. Indeed, if on the contrary $\tilde{a}^{\prime \prime}, b^{\prime \prime}$ are not bounded, then, passing to subsequences and using Proposition 3, one would obtain subsequences $\tilde{a}^{\prime \prime \prime}, b^{\prime \prime \prime}$ such that

$$
E_{0}^{q}\left(\tilde{a}^{\prime \prime \prime}\right) \cong E_{\infty}^{q}\left(b^{\prime \prime \prime}\right)
$$

and either $\tilde{a}^{\prime \prime \prime} \rightarrow \infty$ or $b^{\prime \prime \prime} \rightarrow \infty$. This is impossible because then any operator from $E_{0}^{q}\left(\tilde{a}^{\prime \prime \prime}\right)$ to $E_{\infty}^{q}\left(b^{\prime \prime \prime}\right)$ would be compact. Thus the spaces $E_{0}^{q}\left(\tilde{a}^{\prime \prime}\right)$ and $E_{\infty}^{q}\left(b^{\prime \prime}\right)$ are either finite dimensional, or they are isomorphic to $\ell^{q}$.

Corollary 3. Under the assumptions of Theorem 3, it follows from Proposition 4:

in the case $(\mathrm{A})$ if $p \neq q$ the spaces $E_{0}^{p}(a), E_{0}^{q}(\tilde{a})$ are nuclear, respectively if $q \neq \tilde{q}$ the spaces $E_{\infty}^{q}(b), E_{\infty}^{\tilde{q}}(\tilde{b})$ are nuclear;

in the case $(\mathrm{B})$ if $p \neq q$ then the space $E_{0}^{p}(a)$ is nuclear, respectively if $q \neq \tilde{q}$ then the space $E_{\infty}^{\tilde{q}}(\tilde{b})$ is nuclear.

Using Mityagin's criterion for isomorphisms of power series spaces [12] one can easily obtain from Theorem 2 and Theorem 3 a complete characterization of isomorphisms

$$
E_{0}^{p}(a) \times E_{\infty}^{q}(b) \cong E_{0}^{\tilde{p}}(\tilde{a}) \times E_{\infty}^{\tilde{q}}(\tilde{b}), \quad p \neq \tilde{q} \text { or } q \neq \tilde{p}
$$

in terms of sequences $a, b, \tilde{a}, \tilde{b}$. In particular we have 
Theorem 4. If $p \neq \tilde{q}$ or $q \neq \tilde{p}$ then the following conditions are equivalent:

(i) $E_{0}^{p}(a) \times E_{\infty}^{q}(b) \cong E_{0}^{\tilde{p}}(\tilde{a}) \times E_{\infty}^{\tilde{q}}(\tilde{b})$;

(ii) $E_{0}^{p}(a) \times E_{\infty}^{q}(b) \stackrel{q d}{\cong} E_{0}^{\tilde{p}}(\tilde{a}) \times E_{\infty}^{\tilde{q}}(\tilde{b})$.

If all the spaces $E_{0}^{p}(a), E_{0}^{\tilde{p}}(\tilde{a}), E_{\infty}^{q}(b), E_{\infty}^{\tilde{q}}(\tilde{b})$ are non-Schwartz we have:

Theorem 5. If each of the sequences $a, b, \tilde{a}, \tilde{b}$ does not tend to $\infty$ and $p \neq \tilde{q}$ or $q \neq \tilde{q} \neq \tilde{p}$ then $E_{0}^{p}(a) \times \underset{q d}{q}(b) \cong E_{0}^{\tilde{p}}(\tilde{a}) \times E_{\infty}^{\tilde{q}}(\tilde{b})$ if and only if $\tilde{p}=p, \tilde{q}=q$ and $E_{0}^{p}(a) \stackrel{q d}{\cong} E_{0}^{p}(\tilde{a})$, $E_{\infty}^{q}(b) \stackrel{q d}{\cong} E_{\infty}^{q}(\tilde{b})$.

Let us note that the method used here does not work if $p=\tilde{q}$ and $q=\tilde{p}$. Analogs of Theorem 3 and Theorem 4 in the case $p=q=\tilde{p}=\tilde{q}=1$ were obtained in [2], [3] by using the method of generalized linear topological invariants.

Finally let us note that our approach can be used to obtain analogous results for isomorphic classification of spaces $K^{p}\left(a_{i k}\right) \times K^{q}\left(b_{i k}\right)$, where $\left(a_{i k}\right)$ is $\left(\mathrm{d}_{2}\right)$-matrix and $\left(b_{i k}\right)$ is $\left(\mathrm{d}_{1}\right)$-matrix.

\section{References}

[1] C. Bessaga, Some remarks on Dragilev's theorem. Studia Math. 31, 307-318 (1968).

[2] P. B. Djakov, M. Yurdakul and V. P. Zahariuta, On Cartesian products of Köthe spaces. Bull. Polish. Acad. Sci. Math. 43, $113-117$ (1995).

[3] P. B. DJakov, M. Yurdakul and V. P. Zahariuta, Isomorphic classification of Cartesian products of power series spaces. Michigan Math. J. 43, $221-229$ (1996).

[4] P. B. DJAKov and M. Yurdakul, Strictly singular operators and isomorphisms of Cartesian products. C. R. Acad. Bulgare Sci. 49, No. 4, 9-11 (1996).

[5] M. M. Dragilev, On regular bases in nuclear spaces (in Russian). Mat. Sb. 68, 153-173 (1965).

[6] V. P. Kondakov, On the structure of unconditional bases in some Köthe spaces (in Russian). Studia Math. 76, 137-151 (1983).

[7] J. Lindenstrauss and L. Tzafriri, Classical Banach Spaces I, II. Berlin-Heidelberg-New York 1977, 1979.

[8] B. S. Mityagin, Approximative dimension and bases in nuclear spaces (in Russian). Uzpek. Mat. Zh. 16, 63 -132 (1961). English Transl.: Russian Math. Surveys 16, 59-127 (1961).

[9] B. S. Mityagin and G. M. Henkin, Linear Problems of Complex analysis (in Russian). Uspekhi Mat. Nauk 26, 93 -152 (1971). English Transl.: Russian Math. Surveys 26, 99-164 (1971).

[10] B. S. Mityagin, Equivalence of Bases in the Hilbert Scales (in Russian). Studia Math. 37, 111-137 $(1970-71)$.

[11] B. S. Mityagin, The structure of the infinite Hilbert scale subspace (in Russian). In: Theory of Operators in Linear Spaces, Proceedings of the 7-th Drogobych Mathematical School, 1974, CEMI AS of the USSR, B. Mityagin, ed., 127-133, 1976.

[12] B. S. Mityagin, Non-Schwartzian power series spaces. Math.Z. 182, 303-310 (1983).

[13] S. ÖnAl, T. TERZIOĞLU and M. YuRdaKUl, Isomorphisms of Cartesian products of locally convex spaces. Preprint.

[14] D. Vogt, Sequence space representation of spaces of test functions and distributions. In: Functional Analysis, holomorphy and approximation, G. I. Zapata, ed., Lecture Notes in Pure and Appl. Math. 83, 405 - 443. New York-Basel 1983.

[15] D. Vogt, Frécheträume, zwischen denen jede stetige lineare Abdildung beschränkt ist. J. Reine Angew. Math. 345, 182-200 (1983).

[16] V. V. Wrobel, Streng singuläre Operatoren in lokalkonvexen Räumen. Math. Nachr. 83, 127-142 (1978). 
[17] V. V. WROBEL, Strikt singuläre Operatoren in lokalkonvexen Räumen II, Beschränkte Operatoren. Math. Nachr. 110, 205-213 (1983).

[18] V. P. Zahariuta, The isomorphism and quasiequivalence of bases in Köthe power spaces (in Russian). In: Operator theory in linear spaces, Proceedings of 7-th Winter School in Drogobych, B. Mityagin, ed., CEMI AS of the USSR, 101-126. Moskow 1976.

[19] V. P. ZAHARIUTA, On isomorphisms of Cartesian products of linear topological spaces (in Russian). Funct. Anal. i ego Pril. 4, 87-88 (1970).

[20] V. P. Zahariuta, On the isomorphism of Cartesian products of locally convex spaces. Studia Math. 46, $201-221$ (1973).

[21] V. P. ZAhariutA, Compact operators and isomorphisms of Köthe spaces (in Russian). Aktualnye voprosy matem. analiza, $62-71$ (1978).

[22] V. P. ZAHARIUTA, Linear topologic invariants and their applications to isomorphic classification of generalized power spaces. Tr. J. Math. 20, 237-289 (1996).

Eingegangen am 18. 3. 1996*)

Anschriften der Autoren:

Plamen Borissov Djakov

Department of Mathematics

Sofia University

1164 Sofia

Bulgaria
Süleyman Önal, Murat Yurdakul

Department of Mathematics

Middle East Technical University

06531 Ankara

Turkey
Tosun Terzioğlu Sabanci University Istanbul

Turkey

*) Die vorliegende Fassung ging am 16. 6. 1997 ein. 